\title{
Polysèmes
}

Revue d'études intertextuelles et intermédiales

\section{Correspondances baudelairiennes entre couleur et littérature chez Joris-Karl Huysmans}

Baudelairian Correspondences between Colour and Literature in Huysmans's Writings

\section{Aude Jeannerod}

\section{OpenEdition}

Journals

Édition électronique

URL : https://journals.openedition.org/polysemes/440

DOI : $10.4000 /$ polysemes.440

ISSN : 2496-4212

Éditeur

SAIT

Référence électronique

Aude Jeannerod, « Correspondances baudelairiennes entre couleur et littérature chez Joris-Kar Huysmans ", Polysèmes [En ligne], 14 | 2015, mis en ligne le 18 novembre 2015, consulté le 28 juin 2022. URL : http://journals.openedition.org/polysemes/440 ; DOI : https://doi.org/10.4000/ polysemes. 440

Ce document a été généré automatiquement le 9 octobre 2020.

Polysèmes 


\title{
Correspondances baudelairiennes entre couleur et littérature chez Joris-Karl Huysmans
}

\author{
Baudelairian Correspondences between Colour and Literature in Huysmans's \\ Writings
}

Aude Jeannerod

1 Dans le poème des Fleurs du mal intitulé «Correspondances ", Baudelaire établit une analogie entre les sensations :

Comme de longs échos qui de loin se confondent [...]

Les parfums, les couleurs et les sons se répondent. (Baudelaire I, 11)

Par la synesthésie sont associées différentes perceptions sensorielles: olfactives, auditives et visuelles. De cette analogie découle une théorie de la réception qui se trouve approfondie en 1861 dans Richard Wagner et Tannhäuser à Paris : «ce qui serait vraiment surprenant, c'est que le son ne pût pas suggérer la couleur, que les couleurs ne pussent pas donner l'idée d'une mélodie, et que le son et la couleur fussent impropres à traduire des idées " (II, 784). La théorie des correspondances baudelairiennes fait ainsi subir une mutation d'importance à la doctrine de l'ut pictura poesis : d'une comparaison entre les arts sœurs fondée sur leur pratique, leurs moyens et leurs fins, l'on passe à une similitude dans les effets produits sur le récepteur, qu'il soit lecteur, auditeur ou spectateur. Ainsi, selon Baudelaire dans son Salon de 1846: "La peinture [...] ne ressemble à la poésie qu'autant que celle-ci éveille dans le lecteur des idées de peinture» (II, 474). L'analogie ne porte donc pas sur la conception mais sur la réception:

[...] contrairement à la comparaison qui s'applique aux arts eux-mêmes, qui consiste toujours à comparer ce qu'on appelait leur essence ou leur définition, la correspondance baudelairienne se rapporte uniquement à leurs effets. Elle instaure entre les arts une toute autre forme de relation fondée sur la nature analogue des rêveries qu'ils éveillent, des idées qu'ils suggèrent, des sensations qu'ils excitent chez l'auditeur, le lecteur ou le spectateur. [...]

Saisir des correspondances ne consiste donc pas à comparer des objets, mais à 
comparer les idées et les sensations que ces objets font naître, c'est-à-dire à les

rapprocher uniquement du point de vue du sujet. (Lichtenstein 153)

Et de cette théorie des correspondances procède la définition de la critique d'art donnée par Baudelaire : « le meilleur compte rendu d'un tableau pourra être un sonnet ou une élégie » (II, 418).

Dans son œuvre, l'écrivain et critique d'art Joris-Karl Huysmans veut mettre en pratique ces correspondances: "Je crois à une correspondance entre les vocables de la littérature et les autres arts. Je crois que les transpositions d'un art dans un autre sont possibles » (lettre à Marcel Batilliat, septembre 1891). Ainsi, dans son seul article de critique musicale, "L'Ouverture de Tannhäuser » publié en 1885, il dresse une équivalence entre audition musicale et vision picturale, prenant au pied de la lettre le terme tableau qui désigne chaque moment de l'œuvre de Wagner. Si le «Chant des Pèlerins » a pour équivalent pictural un paysage « où le soleil s'apâlit jusqu'à l'exquise et suprême dilution du jaune d'or, [...] où sous un ciel maladivement lumineux, les montagnes opalisent au-dessus des bleuâtres vallons le blanc cristallisé de leurs cimes » (Huysmans 1994, 198), le "Chant de la Luxure » évoque au contraire une orgie aux accents rubénistes: "l'orchestre éclate, peignant en quelques traits décisifs, [...] Tannhäuser qui s'avance » (200) et « ces grands cuivres [...] semblent une transposition des aveuglantes pourpres et des somptueux ors» (203). Enfin, le retour du thème religieux, qui reprend le pas sur le thème voluptueux, suscite la vision d'une aube incandescente : "le ciel hésitant blanchit comme peint avec des sons blancs de harpe, se teint de couleurs encore tâtonnantes qui peu à peu se décident et resplendissent » (203). Autrement dit, par le biais d'une synesthésie entre vue et ouïe, d'une correspondance entre son et couleur, le compte rendu d'un concert pourra être un tableau.

3 De même, dans sa critique d'art, Huysmans cherche à établir des analogies entre peinture et littérature afin de rendre compte de ce qu'il ressent à la vue d'une œuvre picturale. Selon lui, la tâche du critique est de « surtout décrire le tableau de telle façon que celui qui en lit la traduction écrite, le voie » (Huysmans 2006, 501); mais ce projet se heurte au problème de la couleur et de sa difficile verbalisation. Aussi Huysmans cherche-t-il pour chaque ton un équivalent dans la littérature afin de le faire voir au lecteur : la métaphore littéraire s'impose comme un médium nécessaire pour verbaliser le coloris. Nous verrons ainsi que les œuvres d'art qui retiennent son attention sont celles qui suscitent en lui les mêmes sentiments que ceux qu'il éprouve à la lecture de ses auteurs de prédilection, au premier rang desquels figure Baudelaire, et qu'il décèle surtout ces correspondances dans les genres du paysage et du portrait. Mais nous verrons également qu'à travers la figure de Salomé, il élabore une poétique où les différents arts "se répondent » et « se confondent " (Baudelaire I, 11); il mène ainsi une réflexion interesthétique sur la représentation de l'irreprésentable, sur l'invisible rendu visible dans la peinture et l'illisible rendu lisible dans l'écriture. L'impuissance de chaque médium semble devoir être conjurée par l'analogie avec l'autre.

\section{La peinture de paysage}

4 En 1876, Huysmans consacre un article au peintre Narcisse Diaz de la Peña, récemment décédé. L'éloge funèbre de «ce fanatique de la couleur », "cet enfant gâté de la couleur » (Huysmans 2006, 72) qui fut l'« un des plus remarquables paysagistes » (71), 
se tempère néanmoins de réserves; ce sont précisément les tons de sa palette qui gênent le critique, car la nature peinte par Diaz semble toujours idyllique, voire édénique :

Pour lui, la nature n'a jamais connu le linceul des neiges et le voile brumeux des pluies! La nature, telle qu'il l'a rendue, c'est un blutage de poudre d'or au travers d'une dentelle verte de feuillées, c'est une envolée de lumière sur les velours des mousses. Ni la sérénité des soirs d'août, ni les levers pluvieux de novembre, ni le trouble des bois, la nuit, ni l'alerte du réveil le matin, ni la désolation de l'hiver, ne l'ont tenté. (72)

Le critique ne ressent aucune émotion face à " une éternelle fête, une kermesse de soleil, une liesse de beaux jours » (72) tandis qu'il se trouve ému par les sites désolés et maussades, tel celui qu'il décrit dans «La Bièvre » en $1877:$ : j'avoue ne pas éprouver devant [d]es ripailles de sève, ce charme apitoyé que font naître en moi un coin désolé de grande ville, une butte écorchée, une rigole d'eau qui pleure entre deux arbres grêles » $(1994,109)$. De même, il reproche à l'orientaliste Félix Ziem sa représentation d'une nature toujours ensoleillée et l'éclat de ses couleurs vives:

Jamais de temps couverts, jamais de pluie, jamais de mélancoliques crépuscules avec M. Ziem; et c'est ici qu'il se rattache bien au mouvement de 1830 qui voulait du pittoresque à tout prix et n'admettait la nature qu'à certaines époques, qu'à certaines heures. L'or et la pourpre des couchants étaient fort prisés ; l'insolation de l'art primait tout, aussi le jaune d'œuf et la laque carminée coulent-ils à flots dans les toiles de M. Ziem. (2006, 261-262)

Huysmans associe au romantisme le chatoiement des couleurs, ce qui n'est pas sans rappeler la définition de «l'Écran romantique » élaborée par Émile Zola: «L'Écran romantique est une glace [...] colorée des sept nuances de l'arc-en-ciel. Non seulement elle laisse passer les couleurs, mais elle leur donne encore plus de force» (Zola 378). Aussi Huysmans fait-il de la peinture de Ziem l'équivalent de la poésie de Victor Hugo :

M. Félix Ziem appartient à cette génération d'artistes qui, grisée de couleurs par le romantisme, cingla à toutes voiles vers les Eldorados inconnus du vieil Orient. Aussi faut-il se reporter à l'évolution déterminée par cette école pour bien comprendre le talent de M. Ziem.

L'Orient était à la mode; les poésies d'Hugo, avec leurs fanfares de rimes, leurs vifs bariolages de teintes, leurs éclats fracassants de tons, dominaient, hantant les oreilles et les yeux des poètes et des peintres. (Huysmans 2006, 260)

Les correspondances établies entre ouïe et vue, entre son et couleur nourrissent l'analogie entre poésie et littérature ; et aux "vifs bariolages» des Orientales d'Hugo, Huysmans préfère la douce pâleur des Fleurs du mal. En effet, il fait grief à Charles Daubigny de ne pas se servir de la palette baudelairienne : «Quant à M. Daubigny, son Lever de lune ne me verse pas le pâle enchantement dont parle Baudelaire » (96-97). Car le nocturne bucolique du peintre est bien loin du «Paysage » urbain composé par le poète et intégré aux « Tableaux parisiens » :

Il est doux, à travers les brumes, de voir naître

L'étoile dans l'azur, la lampe à la fenêtre,

Les fleuves de charbon monter au firmament

Et la lune verser son pâle enchantement. (I, 82) 


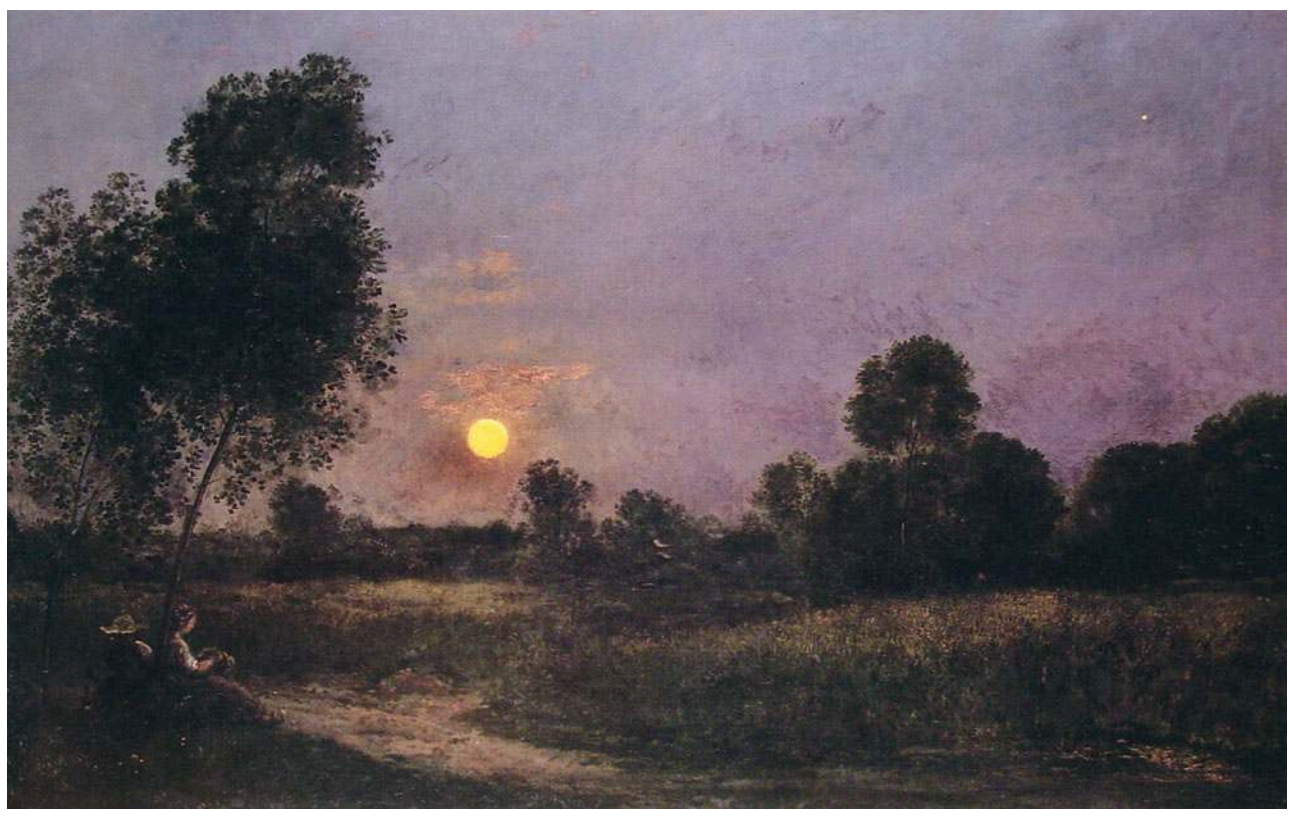

Charles-François Daubigny, Lever de lune (1877), huile sur toile, 134×227cm, coll. part., public domain

La recherche d'un équivalent pictural de la poésie baudelairienne hante tous les écrits huysmansiens sur la peinture de paysage. Ainsi, ce que le critique reproche à Diaz, c'est précisément de ne pas peindre l'automne comme Baudelaire l'écrit : «Si Diaz a parfois aimé l'automne, il n'a jamais su en dégager cette tristesse profonde qui fit écrire à Charles Baudelaire en tête du Confiteor de l'artiste: "Les fins des journées d'automne sont pénétrantes, ah! pénétrantes jusqu'à la douleur! (Huysmans 2006, 72). De même, au Salon, Huysmans déplore l'absence de peintres capables d'égaler le poète : « en est-il un qui sache exprimer la tristesse infinie qui tombe avec le crépuscule, un qui entende cet admirable cri jeté par Baudelaire en tête du Confiteor de l'artiste?» (94). Car l'automne baudelairien n'est pas seulement une saison, c'est également le temps des déceptions et des dépressions :

[Baudelaire] avait révélé la psychologie morbide de l'esprit qui a atteint l'octobre de ses sensations; raconté les symptômes des âmes requises par la douleur, privilégiées par le spleen; montré la carie grandissante des impressions, alors que les enthousiasmes, les croyances de la jeunesse sont taris, alors qu'il ne reste plus que l'aride souvenir des misères supportées, des intolérances subies, des froissements encourus, par des intelligences qu'opprime un sort absurde.

Il avait suivi toutes les phases de ce lamentable automne, regardant la créature humaine, docile à s'aigrir, habile à se frauder, obligeant ses pensées à tricher entre elles, pour mieux souffrir, gâtant d'avance, grâce à l'analyse et à l'observation, toute joie possible. (Huysmans 2004, 175)

Et à la ressemblance du poète de "Brumes et pluies " ${ }^{1}$, Huysmans se déclare touché par les paysages sombres et pluvieux qui semblent faire écho à sa propre mélancolie. Sa description du Froid octobre de John Everett Millais recourt ainsi à un vocabulaire psychologique qui attribue angoisse et spleen au paysage :

[...] la nature attend, désespérée, l'assaut. - Cela est vraiment d'un grand effet cette eau fouettée, ce coup de vent qui fauche les nuées, et va tordre les arbres, cette détresse infinie des automnes qui meurent, ce grand frisson de la nature aux approches des ouragans et des pluies, tout cela est rendu avec une sincérité et une force vraiment admirables. Je n'ai vu, depuis les œuvres si mélancoliques du grand Ruysdael, aucun paysage qui dégage comme celui-ci, une impression de douleur aussi poignante. $(2006,101-102)$ 


\section{Image 100002010000094C0000069316DF5D37.png}

John Everett Millais, Chill October (1870), huile sur toile, $141 \times 187 \mathrm{~cm}$, coll. part., public domain

De même, Huysmans se dit touché par le ciel pluvieux dans Le Moulin de Jacob van Ruysdael :

Jamais paysage ne m'a ainsi ému, et il est fait avec rien! Un moulin à vent égratigne le ciel de ses bras et détache sa carcasse claire sur une tempête de nuages qui roulent et se bousculent, gonflés de pluie! Jamais la mélancolie du grand maître, ravivée encore par le navrement de la nature aux approches des sombres journées d'hiver, n'a été ainsi rendue! Jamais les angoisses de l'artiste qui s'attaque à la nature; jamais la douleur d'un site qui frissonne et se plaint, aux arrivées des neiges, n'a ainsi crié, n'a ainsi saigné dans une toile ! (2006, 74-75)

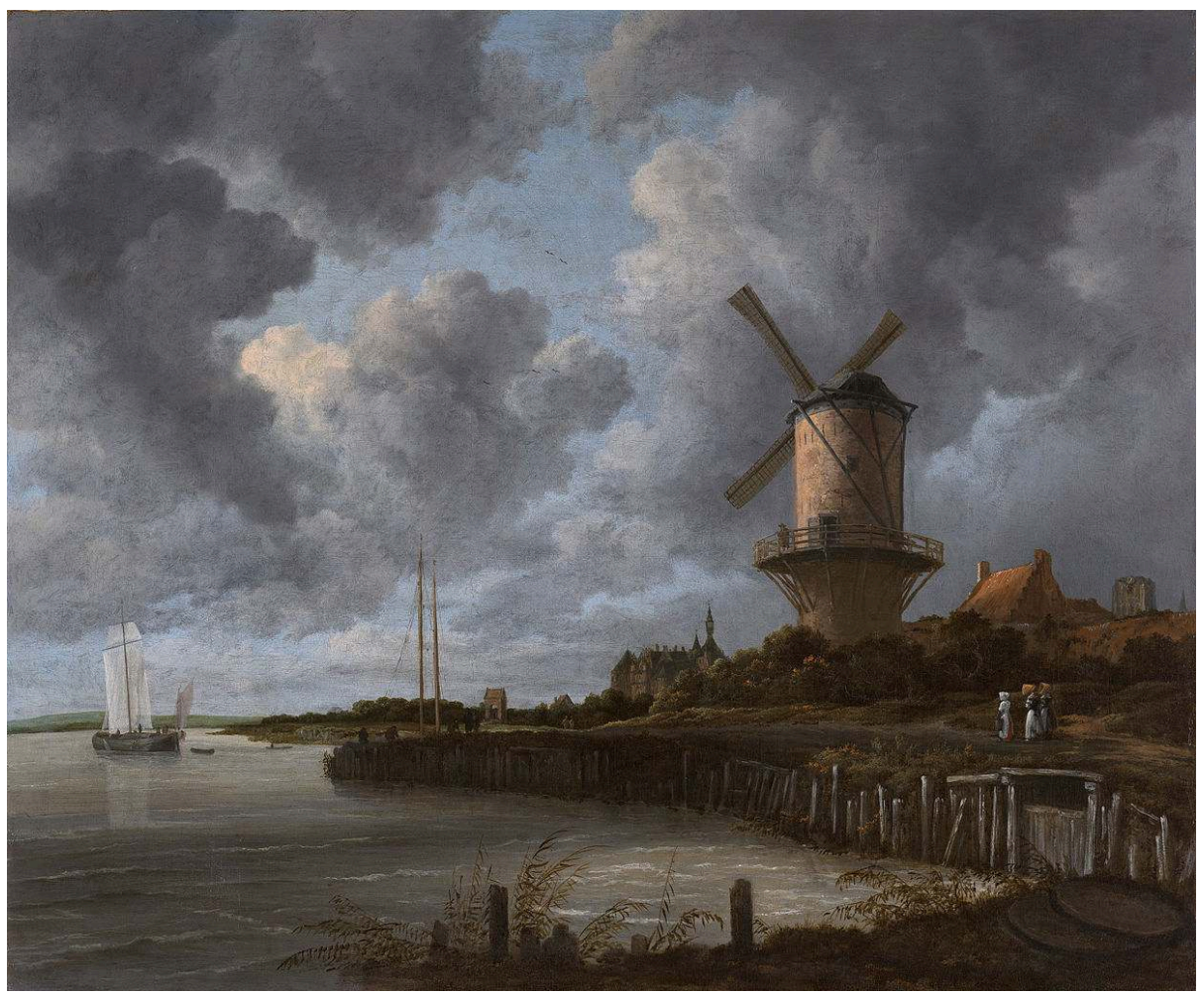

Jacob van Ruysdael, Le Moulin de Wijk-bij-Duurstede (c. 1670), huile sur toile, 83×101cm, Amsterdam, Rijksmuseum, Creative Commons

Les conditions météorologiques confèrent ainsi au paysage une humeur métaphorique, comme Baudelaire, dans "Ciel brouillé ", associe la tension des nerfs à la tension de l'atmosphère orageuse, les dépressions nerveuses aux dépressions atmosphériques :

Tu rappelles ces jours blancs, tièdes et voilés,

Qui font se fondre en pleurs les cœurs ensorcelés,

Quand, agités d'un mal inconnu qui les tord,

Les nerfs trop éveillés raillent l'esprit qui dort. (I, 49)

Ainsi, parce qu'il recherche des œuvres qui le touchent autant que l'émeut la poésie baudelairienne, le critique fait l'éloge de la mélancolie en peinture: «Je ne connais guère de grands paysagistes qui vous fassent éprouver devant leurs toiles une impression rieuse et légère. Rousseau, Millet, Constable et, en prenant dans les anciens, Ruysdael, ont peint des paysages d'où se dégage une grandeur triste. On pourrait dire que la beauté d'un paysage est surtout faite de mélancolie» (Huysmans 2006, 128). La mélancolie est tout autant dans le pinceau de l'artiste que dans le paysage qu'il choisit de peindre : cette correspondance entre état d'âme et état du monde est le fruit d'un 
accord recherché entre l'homme et la nature, que certains peintres de talent parviennent à réaliser.

Jean-François Raffaëlli est sans doute le peintre qui, aux yeux de Huysmans, est le plus à même de traduire sur la toile la mélancolie baudelairienne : « Enfin l'artiste est donc venu, qui aura rendu la mélancolique grandeur des sites anémiques couchés sous l'infini des ciels; voici donc enfin exprimée cette note poignante du spleen des paysages, des plaintives délices de nos banlieues!» (171). Comme le signale l'expression "spleen des paysages », Raffaëlli peint « la mélancolie des jours couverts et des temps pâles » (249) caractéristique du spleen dans Les Fleurs du mal :

Quand le ciel bas et lourd pèse comme un couvercle

Sur l'esprit gémissant en proie aux longs ennuis,

Et que de l'horizon embrassant tout le cercle

II nous verse un jour noir plus triste que les nuits (I, 74)

Huysmans évoque «la mélancolie coutumière » $(2006,173)$ de Raffaëlli, «qu'auront imprégné certaines mélancolies d'humanité et de nature demeurées rebelles, jusqu’à ce jour, à tous les peintres » (250). Il est " le peintre des pauvres gens et des grands ciels » (156), car comme Baudelaire dans les "Tableaux parisiens", il peint la mélancolie urbaine à travers la représentation de la ville et de ses habitants :

Le crépuscule est venu. Dans l'un de ces mélancoliques paysages qui s'étendent autour du Paris pauvre, des cheminées d'usine crachent sur un ciel livide des bouillons de suie. Trois chiffonniers retournent au gîte, accompagnés de leurs chiens. Deux se traînent péniblement, le cachemire d'osier sur le dos et le 7 en main; le troisième les précède, courbé sous la charge d'un sac. (Huysmans 2006, 138)

La désolation du paysage industriel rappelle les « tuyaux » et les " fleuves de charbon » qui se détachent sur les «grands ciels » dans « Paysage » (Baudelaire I, 82), tandis que le défilé des loqueteux évoque le souvenir des «Sept vieillards» (87-88). Et la toile de Raffaëlli suscite chez le spectateur douleur et mélancolie: "J'ai vu au Salon peu de tableaux qui m'aient aussi douloureusement et aussi délicieusement poigné. M. Raffaëlli a évoqué en moi le charme attristé des cabanes branlantes, des grêles peupliers en vedette sur ces interminables routes qui se perdent, au sortir des remparts, dans le ciel » (Huysmans 2006, 138).

Il y a un autre peintre que le critique ne peut jamais évoquer sans que cela suscite en lui le souvenir de la poésie baudelairienne : il s'agit de James Whistler. De ses Nocturnes, Huysmans fait une description qui met en avant les talents de coloriste du peintre :

[...] son délicieux Nocturne en argent et bleu où dans une atmosphère d'azur monte une ville bâtie sur une rive; son Nocturne en noir et or où un feu d'artifices crève de baguettes sanglantes et parsème d'étoiles d'or les ténèbres d'une épaisse nuit, et enfin son Nocturne en bleu et or représentant une vue de la Tamise au-dessus de laquelle, dans une féerique brume, une lune d'or monte, éclairant de ses pâles rayons la forme vague des vaisseaux endormis, à l'ancre. (284) 


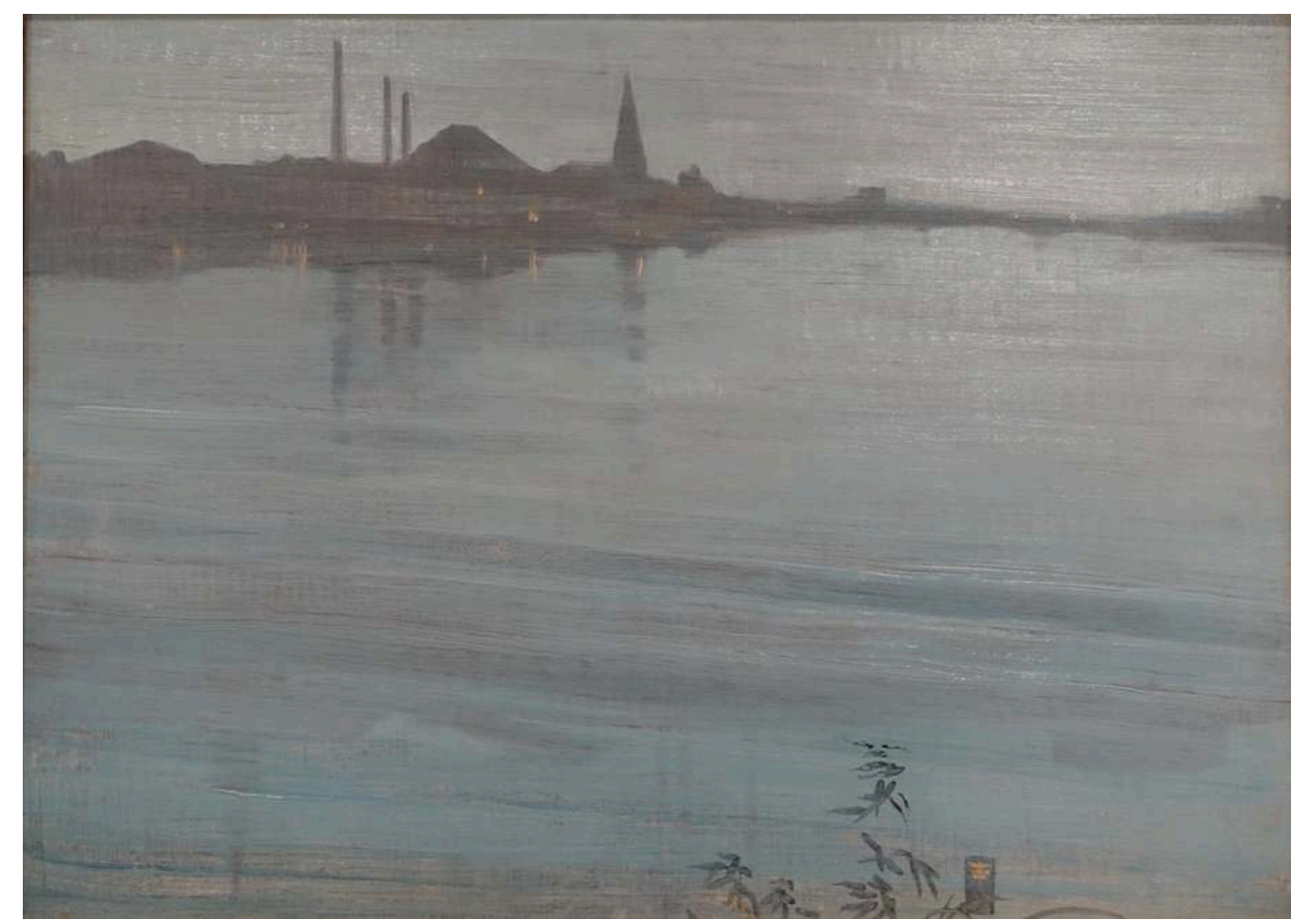

James Abbott McNeill Whistler, Nocturne in Silver and Blue (1871-1872), huile sur toile, 45x61cm, Harvard, Fogg Museum, Creative Commons

\section{Image 10000201000007EE00000A8C0A49603E.png}

James Abbott McNeill Whistler, Nocturne in Black and Gold: The Falling Rocket (1875), huile sur toile, $60 \times 47 \mathrm{~cm}$, Detroit, Institute of Arts, Creative Commons

\section{Image 100002010000177100000F8148CC5187.png}

James Abbott McNeill Whistler, Nocturne in Blue and Gold: Southampton Water (1872), huile sur toile, $51 \times 76 \mathrm{~cm}$, Chicago, The Art Institute, Creative Commons

Ces harmonies et ces contrastes de couleurs font voyager le spectateur vers des contrées apparemment lointaines, celles de la littérature :

Invinciblement, on songeait aux paradis artificiels de De Quincey, à ces vues de rivières, à ces rêves fluides que procure l'opium. Dans leurs cadres d'or blême, vermicelés de bleu-turquoise et piquetés d'argent, des sites d'air et d'eau fuyaient à l'infini, vous transportaient dans un paysage magique et pourtant naturel, évoquaient des au-delà, requéraient des voyages de pensées, suggéraient des dorlotements d'impressions étranges; c'était loin de tout, plus près peut-être de l'art de Baudelaire et d'Edgar Poe que de l'art de la peinture proprement dit. (284)

D'emblée, le rapprochement entre peinture et littérature, invincible, s'impose comme une nécessité. La première source littéraire invoquée est celle de Thomas De Quincey: les couleurs de Whistler suggèrent à Huysmans des hallucinations semblables à celles des Confessions d'un mangeur d'opium anglais (1821). Mais dès les premiers mots, Baudelaire est présent en filigrane, avec l'expression paradis artificiels, car c'est uniquement à travers son adaptation que Huysmans a pris connaissance des visions de De Quincey. De même, Poe et Baudelaire sont étroitement associés, car Huysmans a découvert l'œuvre du premier dans la traduction du second. Les paysages de Whistler outrepassent donc les limites de la peinture pour se rapprocher de la poésie: «en dehors de l'art contemporain " (371), cette peinture ne peut susciter des analogies que littéraires. Les paysages du peintre qui «illimite l'espace, projette des sensations de 
lointains, uniques » (371) évoquent en effet la seconde strophe du poème « Le Poison » dans Les Fleurs du mal:

L'opium agrandit ce qui n'a pas de bornes,

Allonge l'illimité,

Approfondit le temps, creuse la volupté,

Et de plaisirs noirs et mornes

Remplit l'âme au delà de sa capacité. (I, 49)

Mais Huysmans écarte ensuite la référence à Baudelaire afin de lui substituer un poète qui soit plus en accord avec l'art du peintre :

Artiste extra-lucide, dégageant du réel le suprasensible, $\mathrm{M}$. Whistler me fait songer avec ses paysages à plusieurs poésies d'une douceur murmurante et câline, comme confessée, comme frôlée, de M. Verlaine. Il évoque, ainsi que lui, à certains instants, de subtiles suggestions et berce, à d'autres, de même qu'une incantation dont l'occulte sortilège échappe. M. Verlaine est évidemment allé aux confins de la poésie, là où elle s'évapore complètement et où l'art du musicien commence. M. Whistler, dans ses harmonies de nuances, passe presque la frontière de la peinture; il entre dans le pays des lettres, et s'avance sur les mélancoliques rives où les pâles fleurs de M. Verlaine poussent. (Huysmans 2006, 372)

La synesthésie rapproche peinture et littérature à travers la métaphore musicale : la poésie de Verlaine outrepasse les frontières entre les arts au moyen de sons qui suggèrent des couleurs ; de même, la peinture de Whistler fait entendre une musicalité poétique, comme le suggèrent les titres de ses toiles (Arrangement, Harmonie, Symphonie, etc.).

7 Ainsi, Huysmans reconnaît être attiré par la peinture qui suscite chez lui les mêmes émotions que celles qu'il ressent à la lecture de Baudelaire, De Quincey, Verlaine et Poe: mélancolie à la vue des banlieues désolées de Raffaëlli, fascination devant les paysages fantasmatiques de Whistler. Si la peinture de paysage est capable de suggérer un état d'âme, cette dimension psychologique est d'autant plus prégnante dans le genre du portrait.

\section{Le genre du portrait}

L'évolution du naturalisme de Huysmans dans les années 1880 le pousse à se détacher du matérialisme zolien qui se préoccupe davantage du corps que de l'esprit. L'écrivain et critique d'art part alors à la recherche de formes littéraires et picturales capables de représenter l'intériorité, de rendre l'âme humaine. Dans À rebours, des Esseintes trouve ainsi dans la poésie de Baudelaire une manifestation de la vie, non seulement extérieure et physiologique, mais intérieure et psychologique :

Et plus des Esseintes relisait Baudelaire, plus il reconnaissait un indicible charme à cet écrivain qui, dans un temps où le vers ne servait plus qu'à peindre l'aspect extérieur des êtres et des choses, était parvenu à exprimer l'inexprimable, grâce à une langue musculeuse et charnue, qui, plus que toute autre, possédait cette merveilleuse puissance de fixer avec une étrange santé d'expressions, les états morbides les plus fuyants, les plus tremblés, des esprits épuisés et des âmes tristes. (Huysmans 2004, 176)

En s'intéressant à la psyché, Baudelaire dit l'indicible et exprime l'inexprimable. Et si les mots sont réticents à rendre l'énigme de l'âme et de la pensée, la gageure rencontrée par le peintre qui s'y efforce est encore plus grande. En effet, les arts plastiques, par leur matérialité même, rendent difficile la suggestion de l'immatériel: comment représenter une idée, une sensation, un sentiment? Huysmans traque dans les 
expositions de peinture l'artiste qui sera capable de donner à voir l'esprit à travers la matière, l'invisible par le biais du visible. Et les portraits de Whistler répondent pleinement à ces exigences esthétiques; en effet, Huysmans voit dans Thomas Carlyle l'illustration de la dualité humaine : «C'est un portrait qui pénètre sous la peau, met sur la physionomie du personnage un reflet des pensées qu'il médite, un portrait en quelque sorte psychologique» (Huysmans 2006, 285). Le portrait conjoint surface et profondeur ("sous la peau » et "sur la physionomie») en matérialisant les pensées immatérielles du personnage : « c'est un portrait d'âme ouverte » (370).

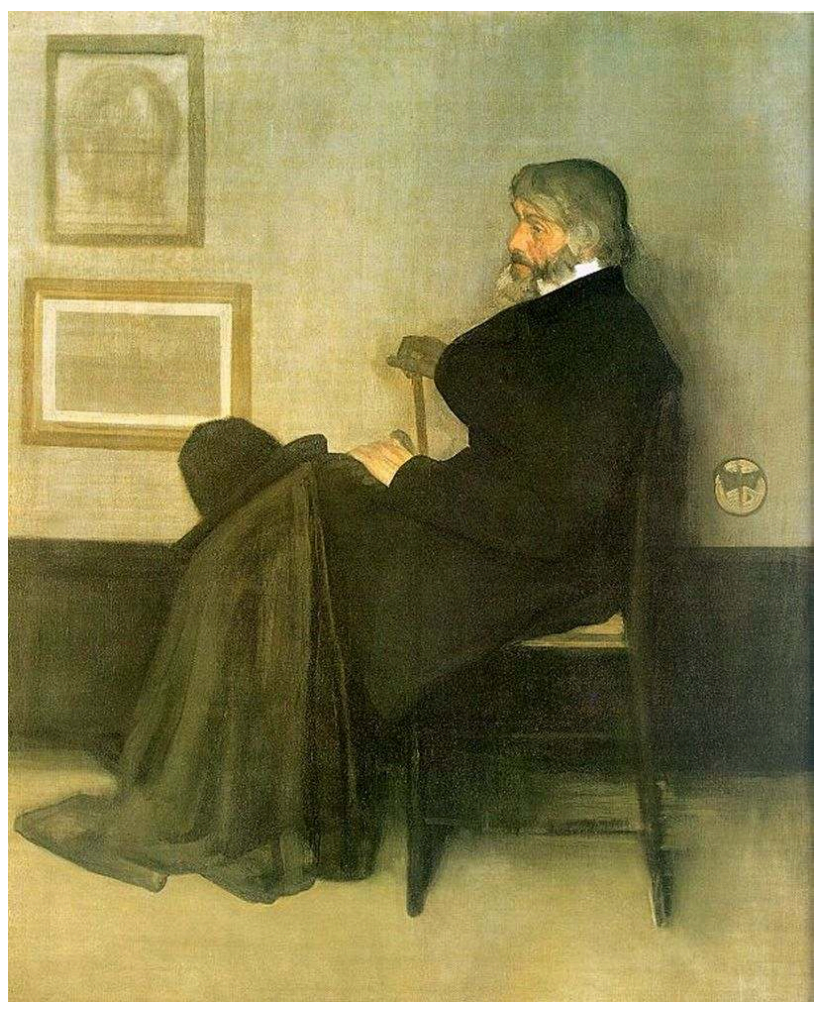

James Abbott McNeill Whistler, Arrangement in Gray and Black n²: Portrait of Thomas Carlyle (1873), huile sur toile, $171 \times 144 \mathrm{~cm}$, Glasgow, Kelkingrove Art Museum, Creative Commons

Huysmans célèbre également la vie double de Lady Campbell, « dont le corps palpite et vit et dont le mystérieux visage, avec son œil énigmatique et sa bouche d'un rouge glacé, inquiète. Cette fois encore, le peintre a sorti de la chair vêtue une expression indéfinie d'âme » (310). Les substantifs insistent sur la matérialité du corps, mais les adjectifs tissent un autre réseau sémantique, qui semble trouver son unité dans le dernier mot : âme. La dualité essentielle des êtres, divisés entre la chair et l'âme, semble être le mystère, l'énigme dont il est ici question. 


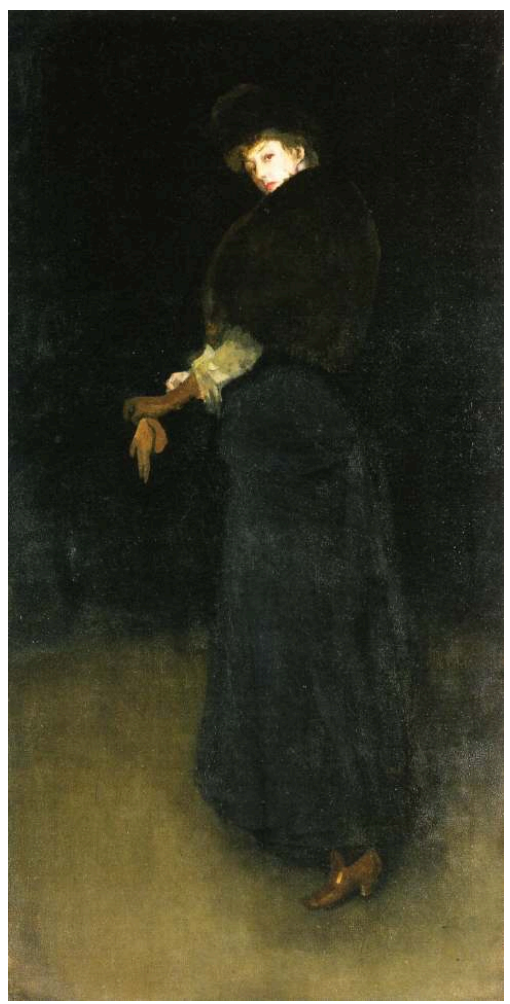

James Abbott McNeill Whistler, Arrangement in Black: The Lady in the Yellow Buskin (1883), huile sur toile, $218 \times 110 \mathrm{~cm}$, Philadelphia, Museum of Art, Creative Commons

Ce dédoublement de l'être entre corps et âme est également rendu visible dans le Portrait de la mère de l'artiste :

C'est inquiétant, mystérieux, d'une couleur différente de celle que nous avons l'habitude de voir. [...] l'accord du gris et du véritable noir de Chine était une joie pour les yeux surpris par ces harmonies inhabituelles; c'était je ne sais quoi, de la peinture anglaise baudelairisée, de la peinture lunaire et réelle. (284)

Ce je ne sais quoi qui déconcerte le spectateur ne peut être exprimé que par un néologisme : «baudelairisé ». Et cet indéfinissable, qui déjà se scinde entre « lunaire et réelle ", se précise encore plus loin : "c'était de la peinture réaliste, toute intime, mais s'éployant déjà dans l'au-delà du rêve " (369). Le mystère auquel Huysmans assiste est celui d'une incarnation profane : ce qui est de l'ordre du psychique se matérialise dans la substance picturale ; l'intangible devient palpable sous la forme d'une œuvre d'art. 


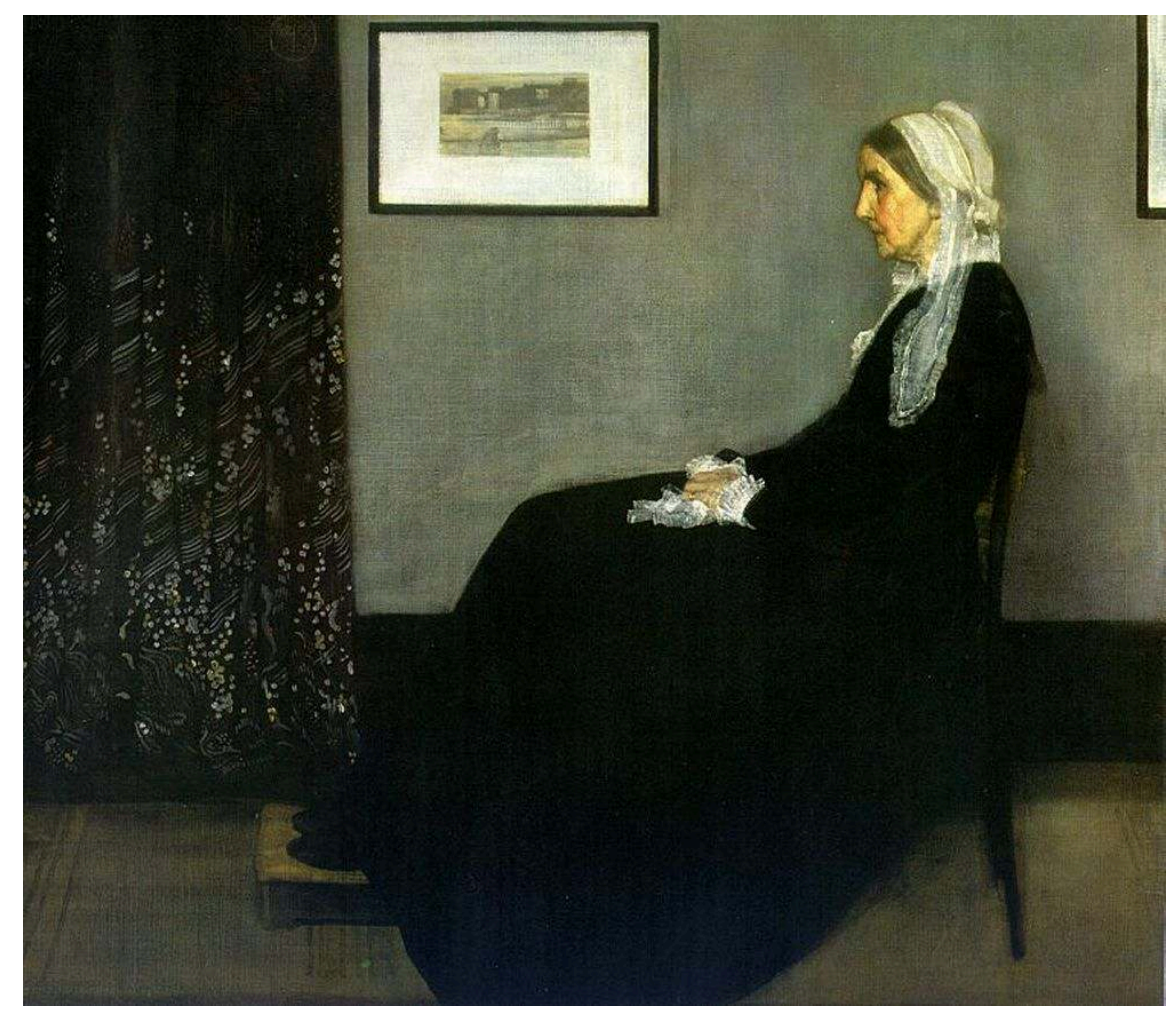

James Abbott McNeill Whistler, Arrangement in Grey and Black: Portrait of the Artist's Mother (1871), huile sur toile, $144 \times 163 \mathrm{~cm}$, Paris, musée d'Orsay, Creative Commons

9 Ainsi, Huysmans donne pour équivalent à la poésie baudelairienne les teintes «baudelairisée[s] " employées par Whistler: «du gris et du véritable noir », une couleur « lunaire » (284) qui rappelle le "pâle enchantement » (96) évoqué plus haut. Il tient Whistler pour un grand coloriste, bien conscient de ce que cette appellation peut avoir de paradoxal à propos d'un peintre qui privilégie les harmonies de gris : "les couleurs sont tristes, comme déteintes. L'on dirait d'un Manet atone» (311) et "sa couleur même, si délicate, paraît évidemment fade aux rétines des bourgeois convaincus que MM. Clairin et Benjamin-Constant sont des coloristes parce qu'à force de souffler, ils faussent les trombones des teintes vives qu'ils appuient jusqu'à les faire éclater, par les cymbales des tons complémentaires " (285). À la fanfare des couleurs vives, Huysmans préfère la mélodie des tons tendres; à propos du Portrait de vieille femme d'Edward Riche Butler, il file la métaphore musicale: «C'est une harmonie whistlerienne de rosâtre et de gris ; de gris, chanté par le ton de la robe et le fond de la toile; de rosâtre modulé par la tête, les mains et le carreau de la chambre» (311). Systématiquement, il signale aussi la faible quantité de matière qui recouvre les toiles de Whistler : sa peinture semble caractérisée par sa ténuité, voire son inexistence. Lady Meux «est peinte avec une légèreté, un superficiel de couleurs » (275), Miss Cicely Alexander "est encore, dans son large fini, peint à peine; la toile est tout juste recouverte " (285), et dans le portrait de sa mère, "la toile était avec cela à peine chargée et montrait presque son grain » (284). Contrairement à l'impressionnisme qui recherche un effet de présence par la matérialité de la couleur, Whistler rend sensible l'âme de ses portraits par une couleur quasi absente: des harmonies de teintes atones et très peu de matière sur la toile. 


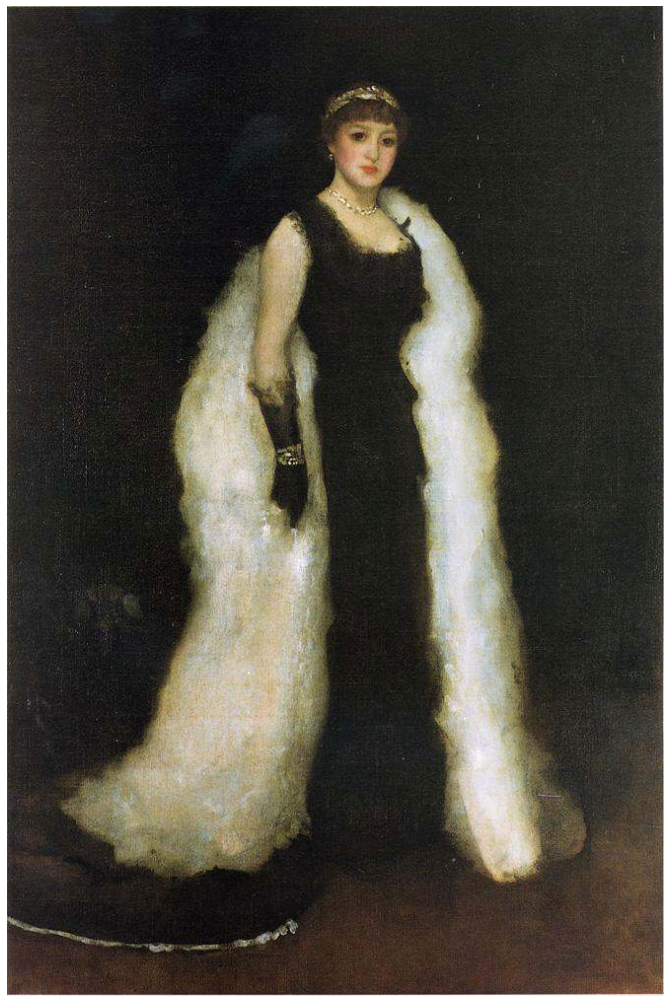

James Abbott McNeill Whistler, Arrangement in Black $n^{\circ} 5$ : Lady Meux (1881), huile sur toile, 194x130cm, Honolulu, Museum of Art, Creative Commons

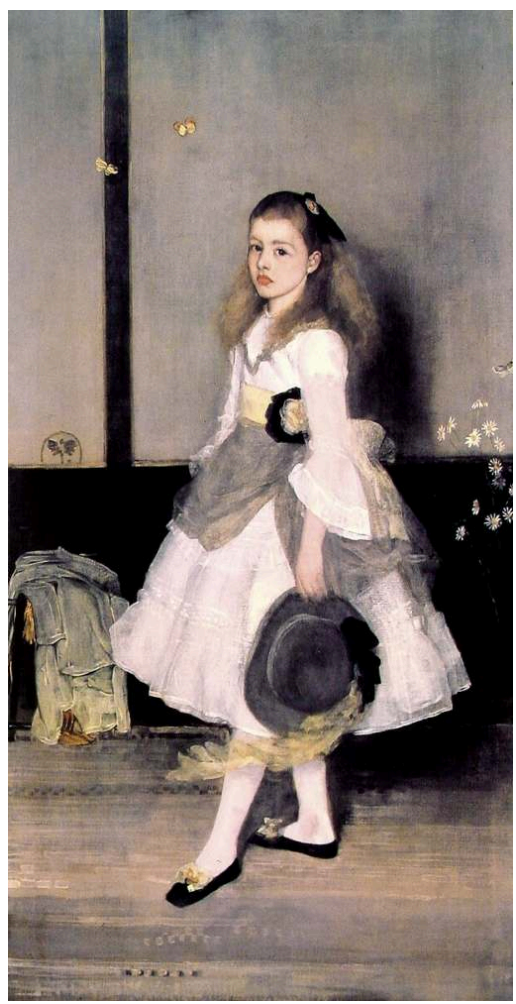

James Abbott McNeill Whistler, Harmony in Grey and Green: Miss Cicely Alexander (1872-1874), huile sur toile, 190x98cm, Londres, Tate Gallery, Creative Commons

Les portraits de Whistler séduisent donc Huysmans parce qu'ils représentent à la fois l'âme et le corps, ou plutôt parce que la physionomie des figures lui laisse toute latitude 
pour se figurer leur état psychologique. Manifestant l'invisible dans le visible, l'art de Whistler est donné comme un équivalent pictural de la poésie de Baudelaire. Mais Huysmans dit ressentir, face à ces toiles, une trouble fascination et une hésitation de la raison qu'il éprouve également à la lecture des contes fantastiques d'Edgar Poe. Selon le critique, l'artiste donne une forme sensible à ce qui se dérobe à toute saisie sensorielle ou rationnelle; Huysmans associe donc les portraits de l'artiste à des visions médiumniques :

L'on ne peut, en effet, lire les révélations plus ou moins véridiques du docteur Crookes sur cette $\mathrm{Katie}^{2}$, sur cette ombre incarnée en une forme dédoublée de femme tangible et pourtant fluide, sans songer à ces portraits de femmes de Whistler, ces portraits-fantômes qui semblent reculer, vouloir s'enfoncer dans le mur, avec leurs yeux énigmatiques et leur bouche d'un rouge glacé, de goule. (2006, 370)

Dans ces œuvres, il voit donc la représentation de visions surnaturelles, d'apparitions spectrales : Lady Meux est « un portrait noir, fantomatique et bizarre » (284) qui « vous attire et vous fascine» (275) et Lady Campbell «fascine et trouble [...] comme une apparition d'un autre monde » (310). Cette hésitation de la raison, Huysmans la ressent également à la lecture de Poe ; aussi établit-il une correspondance entre Miss Cicely Alexander et la nouvelle intitulée Ligeïa, dans laquelle le narrateur opiomane voit apparaître le spectre de sa défunte épouse :

[...] il y a, dans cette toile, un coin supraterrestre, déconcertant. [...] il y a aussi un côté surnaturel émané de ce peintre mystérieux un peu spectral [...]. Il y a un fait certain, c'est que M. Whistler est en dehors de tout l'art contemporain, qu'il a créé une peinture morbide, si l'on veut, mais une peinture toute personnelle, qu'il est en somme, le peintre enchanté des fluides, le thaumaturge qui glisse toujours un peu de l'âme des «Ligeïa » dans ses inquiétants portraits de femmes. (285)

Le spectateur se voit décontenancé par la présence de l'invisible dans le visible, et Whistler étant «en dehors de tout l'art contemporain", le rapprochement avec la littérature semble s'imposer. Or, dans Ligeïa, Poe (et à sa suite Baudelaire) recourt à une analogie avec les paradis artificiels afin de faire voir la beauté du personnage éponyme : "C'était l'éclat d'un rêve d'opium " (Poe 242). Ainsi, au fil des tableaux s'affermit la cohérence du réseau de métaphores littéraires mis en place pour parler de la peinture de Whistler, réunissant Baudelaire, Poe et De Quincey, trois auteurs profondément liés les uns aux autres. Sous la plume de Huysmans, les contes de Poe et les portraits de Whistler deviennent donc indissociables, et ils sont évoqués conjointement lorsque le critique souhaite rendre compte de l'impression surnaturelle qui se dégage d'une toile. C'est d'abord le Chant d'automne de Jacques-Émile Blanche qui éveille leur souvenir: "L'impression est curieuse, un peu fantomatique, presque dolente, toute pâle. Ces figures élancées vêtues de blanc tiennent terre à peine; il $\mathrm{y} a$, en elles, un soupçon de l'énigme que les figures d'Edgar Poe et de M. Whistler laissent entrevoir " (Huysmans 2006, 333). Et quelques lignes plus loin, Whistler sert de nouveau de comparant pour décrire Miss Bury de Frantz Charlet: "peinture captieuse, impression ambiguë, rappelant de lointains Whistler » (333-334). D'une toile à l'autre, d'un artiste à l'autre, c'est toujours le même réseau sémantique qui est à l'œuvre.

11 Ainsi, les portraits de Whistler suscitent chez le spectateur deux réactions complémentaires: d'une part, attrait et fascination; d'autre part, malaise et inquiétude. À propos de Lady Campbell, Huysmans évoque «son œil incitant et hautain qui convie et sa bouche d'un rouge mat qui repousse", faisant d'elle " une inquiétante sphynge » (371) ; la référence à la sphynge convoque la figure de la femme fatale, qui 
séduit et effraye tout à la fois ${ }^{3}$. Mais plus que chez Whistler, c'est surtout chez Gustave Moreau que Huysmans va trouver un équivalent pictural de la femme fatale présente dans la poésie baudelairienne.

\section{La figure de Salomé}

Dans À rebours, des Esseintes possède deux tableaux de Moreau : Salomé dansant devant Hérode et L'Apparition. Pour décrire ces œuvres, Huysmans critique d'art recourt encore une fois au topos de l'indescriptible, qui impose l'analogie avec la littérature : «C'est qu'en effet ses toiles ne semblent plus appartenir à la peinture proprement dite. [...] Cela est plus complexe encore, plus indéfinissable. La seule analogie qu'il pourrait y avoir entre ces œuvres et celles qui ont été créées jusqu'à ce jour n'existerait vraiment qu'en littérature " $(2006,190)$. Aussi des Esseintes accroche-t-il ces œuvres dans sa bibliothèque, comme des livres parmi d'autres, «pendues aux murailles de son cabinet de travail, sur des panneaux réservés entre les rayons des livres» $(2004,96)$. La peinture est traitée comme la littérature, car elle a charge de rendre visible le lisible: dans la plus pure tradition de l'ut pictura poesis, Moreau a traduit les mythes sur la toile, «il les a exprimés dans une langue persuasive et superbe, mystérieuse et neuve » $(2006,346)$. Mais à la référence biblique qui préexiste au pictural, Huysmans substitue une analogie poétique où peinture et littérature entrent en correspondance. En effet, dans le dernier quart du XIX ${ }^{e}$ siècle, Salomé n'est pas seulement l'archétype de la femme fatale, elle est également " une médiatrice esthétique, un lieu où penser l'unité de l'art » (Coste 69), "le carrefour obligé de tous les systèmes sémiotiques [...]: sa simple évocation, par n'importe quel médium, fait se côtoyer danse, musique, tragédie, figuration plastique, récit légendaire et vocation lyrique » (Coste 85). En effet, Salomé, parce qu'elle une danseuse, incarne le modèle d'un art total ; selon Baudelaire : "La danse peut révéler tout ce que la musique recèle de mystérieux, et elle a de plus le mérite d'être humaine et palpable. La danse, c'est la poésie avec des bras et des jambes, c'est la matière, gracieuse et terrible, animée, embellie par le mouvement ». Et le poète ajoute : "Ceux-là seuls peuvent me comprendre à qui la musique donne des idées de peinture » (Baudelaire I, 573). Salomé apparaît donc comme un creuset dans lequel les arts entrent en correspondance, correspondance renforcée par l'abondance d'œuvres musicales, picturales et littéraires qu'elle inspire depuis l'Antiquité, et plus particulièrement dans la deuxième moitié du XIX ${ }^{\mathrm{e}}$ siècle. 


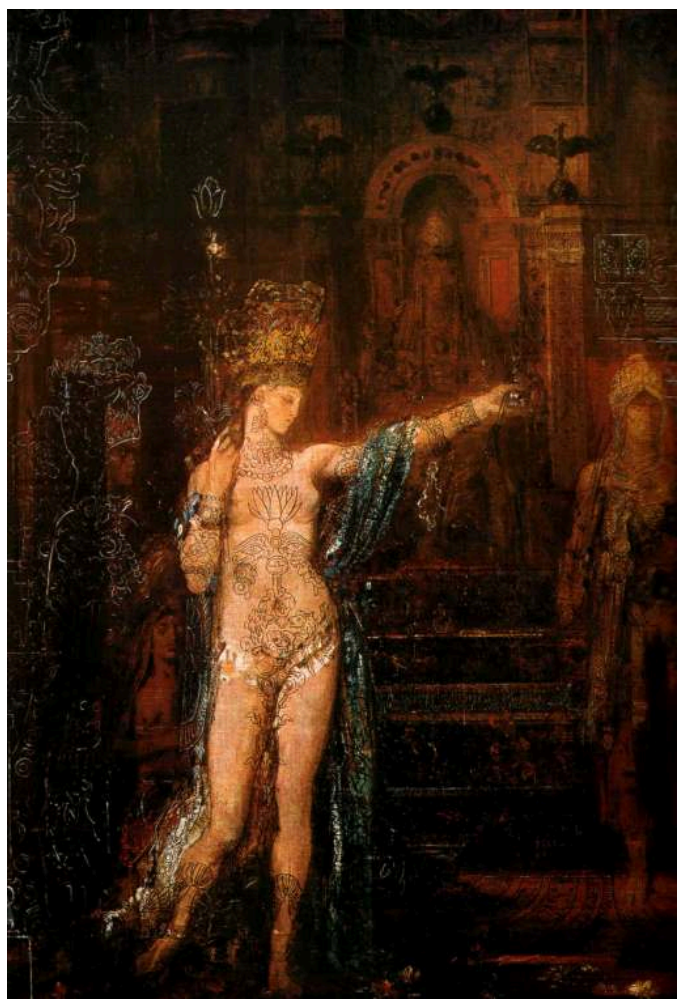

Gustave Moreau, Salomé dansant devant Hérode (1876), huile sur toile, 144x104cm, Los Angeles, Hammer Museum, Creative Commons

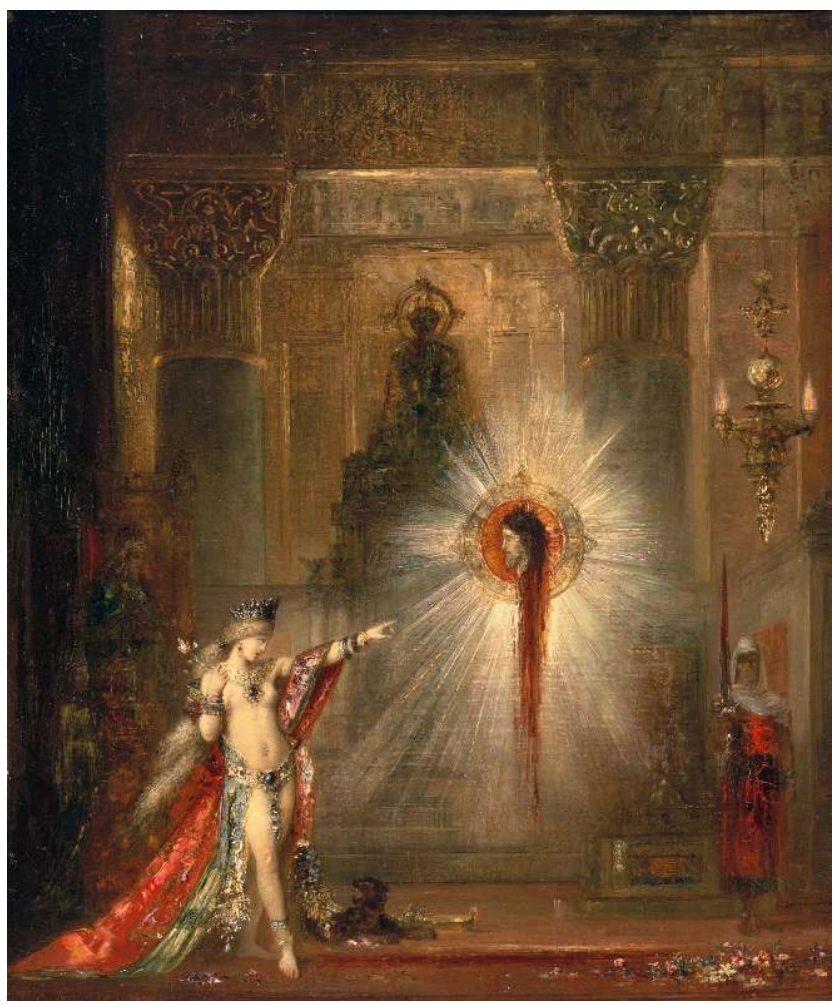

Gustave Moreau, L'Apparition (1876), aquarelle, 106x72cm, Paris, musée d'Orsay, Creative Commons

13 C'est d'abord à la poésie de Baudelaire que l'art de Moreau fait penser : « Il y avait dans ses œuvres désespérées et érudites un enchantement singulier, une incantation vous remuant jusqu'au fond des entrailles, comme celle de certains poèmes de Baudelaire, et 
l'on demeurait ébahi, songeur, déconcerté, par cet art qui franchissait les limites de la peinture, empruntait à l'art d'écrire ses plus subtiles évocations » (Huysmans 2004, 95-96). En effet, à l'ambiguïté propre à la femme dans la poésie baudelairienne répond l'ambivalence de Salomé dans la peinture de Moreau. Ainsi, même si la danseuse n'est pas explicitement nommée dans Les Fleurs du mal, Huysmans y décèle tout de même " une Salomé virtuelle » (Marchal 15) dont il développe les potentialités. En effet, dans sa description de la danse de Salomé "indifférente, irresponsable, insensible " (Huysmans 2004, 92), l'écrivain semble se souvenir du sonnet de Baudelaire décrivant lui aussi une danseuse "Insensible [...] à l'humaine souffrance ", qui "se développe avec indifférence " (I, 29). Et quand Huysmans fait de Salomé «la Beauté maudite » $(2004,92)$ sur le ventre de laquelle «bat une gigantesque pendeloque où coule une rivière d'escarboucles » (94), il se livre à une réécriture de l'« Hymne à la Beauté » :

Tu marches sur des morts, Beauté, dont tu te moques ;

De tes bijoux l'Horreur n'est pas le moins charmant,

Et le Meurtre, parmi tes plus chères breloques,

Sur ton ventre orgueilleux danse amoureusement. (Baudelaire I, 25)

L'allure fantomatique de Salomé, "les yeux fixes, semblable à une somnambule" (Huysmans 2004, 90), convoque également le souvenir d'« Un Fantôme » et de "sa rêveuse allure orientale» (Baudelaire I, 38) - d'autant plus que le texte baudelairien proposait déjà une analogie avec la peinture :

Comme un beau cadre ajoute à la peinture,

Bien qu'elle soit d'un pinceau très vanté,

Je ne sais quoi d'étrange et d'enchanté

En l'isolant de l'immense nature,

Ainsi bijoux, meubles, métaux, dorure,

S'adaptaient juste à sa rare beauté ;

Rien n'offusquait sa parfaite clarté,

Et tout semblait lui servir de bordure. (I, 39)

De plus, la dénudation de la danseuse - «Elle est presque nue ; [...] elle n'est plus vêtue que de matières orfévries et de minéraux lucides » (Huysmans 2004, 93) - rappelle «Les Bijoux » : "La très chère était nue, et, connaissant mon cœur, / Elle n'avait gardé que ses bijoux sonores ». Dans ce poème, Baudelaire invite également à la synesthésie entre ouïe et vue, autrement dit entre poésie et peinture : « j'aime à la fureur / Les choses où le son se mêle à la lumière » (I, 158).

Dans L'Art moderne, Huysmans précise l'analogie en associant l'art de Moreau à un poème en particulier: "L'on éprouve, en effet, devant ces tableaux, une sensation presque égale à celle que l'on ressent lorsqu'on lit certains poèmes bizarres et charmants, tels que le Rêve dédié, dans les Fleurs du Mal, à Constantin Guys, par Charles Baudelaire " $(2006,190)$. Or, dans "Rêve parisien », Baudelaire lui-même se déclare « peintre », créateur d'une «Babel d'escaliers et d'arcades » (I, 101-102) qui trouve son équivalent dans le palais d'Hérode peint par Moreau. Cette architecture féerique entre également en correspondance avec les hallucinations des Paradis artificiels: «D'étonnantes et monstrueuses architectures se dressaient dans son cerveau, semblables à ces constructions mouvantes que l'œil du poète aperçoit dans les nuages colorés par le soleil couchant » (Baudelaire I, 482). Car les tableaux de Moreau relèvent, selon Huysmans, de "ces féeries écloses dans le cerveau d'un mangeur d'opium " $(2006,191)$; et dans À rebours, "sous l'action des Gustave Moreau pendus aux murs ", des Esseintes s'abîme dans des rêveries semblables à celles que connaît "après une touche d'opium, De Quincey» $(2004,116)$. La correspondance établie entre peinture et 
littérature s'éprouve donc du point de vue de la réception : Moreau, comme Baudelaire ou De Quincey, est capable de « rendre ses rêves visibles » $(2006,190)$ pour le lecteurspectateur.

Mais le critique revient ensuite sur cette analogie, par un repentir qui se veut plus exact : «Et encore le style de M. Moreau se rapprocherait-il plutôt de la langue orfévrie des de Goncourt. S'il était possible de s'imaginer l'admirable et définitive Tentation de Gustave Flaubert, écrite par les auteurs de Manette Salomon, peut-être aurait-on l'exacte similitude de l'art si délicieusement raffiné de M. Moreau » (190). D'une part, Huysmans établit une correspondance entre l'écriture artiste des Goncourt et la peinture dite littéraire de Moreau - rapprochement d'autant plus autorisé que les romanciers ont pris pour héroïne une femme qui se révèle fatale à la carrière d'artiste du personnage masculin, et qu'ils ont choisi de la nommer Manette Salomon. D'autre part, la danse de séduction opérée sur Hérode par Salomé convoque le souvenir des tentatrices qui sollicitent l'ascète dans la Tentation de saint Antoine; aussi Huysmans décrit-il la Salomé de Moreau comme " du Flaubert pictural » (lettre à Albert Bartholomé, mars 1883). En miroir, les romans de Flaubert sont assimilés à des peintures : «Chez Flaubert, c'étaient des tableaux solennels et immenses, des pompes grandioses dans le cadre barbare et splendide desquels gravitaient des créatures palpitantes et délicates, mystérieuses et hautaines " (Huysmans 2004, 210). De même, la poésie de Mallarmé produit sur le lecteur une impression quasi visuelle: «il se dispensait d'éparpiller l'attention sur chacune des qualités qu'auraient pu présenter, un à un, les adjectifs placés à la queue leu leu, la concentrait sur un seul mot, sur un tout, produisant, comme pour un tableau par exemple, un aspect unique et complet, un ensemble » (225). L'équivalence est telle que des Esseintes, à la faveur de rêveries nocturnes, voit se réaliser l'idéal d'un art total qui conjoint peinture et poésie, L'Apparition de Moreau faisant entendre l'Hérodiade de Mallarmé :

Combien de soirs, sous la lampe éclairant de ses lueurs baissées la silencieuse chambre, ne s'était-il point senti effleuré par cette Hérodiade qui, dans l'œuvre de Gustave Moreau maintenant envahie par l'ombre, s'effaçait plus légère, ne laissant plus entrevoir qu'une confuse statue, encore blanche, dans un brasier éteint de pierres! [...]

Invinciblement, il levait les yeux vers elle, la discernait à ses contours inoubliés et elle revivait, évoquant sur ses lèvres ces bizarres et doux vers que Mallarmé lui prête. (224)

Salomé est donc bien le lieu où s'unissent les arts, où la poésie fait image, où le tableau prend voix, ces correspondances décuplant le pouvoir expressif et suggestif de chaque médium. En elle se concentrent les modèles picturaux, poétiques et romanesques de Huysmans. Aussi le texte de l'écrivain, qui se confronte lui-même à ces modèles, s'inscrit-il également dans ce réseau de textes et d'images :

Le choix d'écrire une Salomé [...] relève d'abord d'une stratégie littéraire; c'est se confronter à un objet à la fois distant et proche, relevant de l'Histoire sainte et déjà entièrement littérarisé, en face duquel il n'y a pas d'ingénuité possible, et c'est se mettre par conséquent en situation de secondarité délibérée, pour faire mieux, ou autre chose ; c'est jouer le jeu de la réécriture [...]. C'est en tout cas mettre au jour des enjeux intertextuels, intergénériques, interesthétiques, et par là même une dimension critique de l'écriture, à une époque où l'idée même de littérature connaît une profonde transformation. (Marchal 9)

Aucun texte n'ayant pour l'instant été capable de proposer à lui seul un équivalent langagier de la peinture de Moreau, cette gageure ne pouvait manquer de stimuler l'écrivain. Ainsi, dans À rebours, Huysmans propose une réécriture du mythe de Salomé 
qui se réclame tout autant de la peinture de Moreau et de la poésie de Baudelaire et Mallarmé, que de la prose de Flaubert et des Goncourt. Salomé permet donc d'articuler les correspondances baudelairiennes à un nouvel art poétique, et le texte huysmansien « apparaît en fait comme une tentative pour dépasser la vieille opposition entre roman et poésie, et réécrire en une œuvre unique inspirée des Salomés de Moreau La Tentation de saint Antoine et Les Fleurs du mal » (Marchal 193).

Ainsi, pour faire voir la couleur, Huysmans recourt à la métaphore littéraire et se réfère à l'œuvre d'autres écrivains. Il expérimente donc les limites de la critique d'art en tant que genre descriptif : s'il est incapable de rendre compte d'un tableau sans renvoyer à l'écriture d'autrui, c'est parce que le discours critique échoue à entrer en correspondance avec la peinture. Les peintres qu'il admire le plus, les artistes qui le fascinent semblent résister à la simple description. Autrement dit, afin de fournir luimême l'équivalent littéraire d'une œuvre d'art, Huysmans doit prendre ses distances vis-à-vis du genre qu'il pratique: c'est pourquoi ses transpositions d'art s'affranchissent du genre critique afin d'envahir ses romans.

\section{BIBLIOGRAPHIE}

Baudelaire, Charles. Euvres complètes. 2 tomes. Éd. Claude Pichois. Paris : Gallimard, « Bibliothèque de la Pléiade », 1975-1976.

Coste, Didier. « Salomé vue par : programmes narratifs et programmes visuels ». Des mots et des couleurs II. Jean-Pierre Guillerm (dir.). Lille : PU de Lille, 1986, 69-87.

De Quincey, Thomas. CEuvres. Pascal Aquien (ed.). Paris : Gallimard, « Bibliothèque de la Pléiade », 2011.

Dijkstra, Bram. Idols of Perversity : Fantasies of Feminine Evil in Fin-de-Siècle Culture. Oxford: OUP, 1986.

Dottin-Orsini, Mireille. Cette Femme qu'ils disent fatale. Textes et images de la misogynie fin-de-siècle. Paris : Grasset, 1993.

Huysmans, Joris-Karl. À rebours (1884). Daniel Grojnowski (ed.). Paris : Flammarion, « GF », 2004.

Huysmans, Joris-Karl. Croquis parisiens (1886). Paris : La Bibliothèque des Arts, 1994.

Huysmans, Joris-Karl. Écrits sur l'art (1867-1905). Patrice Locmant (ed.). Paris : Bartillat, 2006.

Lichtenstein, Jacqueline. La Tache aveugle. Essai sur les relations de la peinture et de la sculpture à l'âge moderne. Paris : Gallimard, « NRF essais », 2003.

Marchal, Bertrand. Salomé entre vers et prose. Baudelaire, Mallarmé, Flaubert, Huysmans. Paris : José Corti, « Les Essais », 2005.

Poe, Edgar Allan. Cuvres en prose. Trad. Charles Baudelaire. Yves-Gérard Le Dantec (ed.). Paris : Gallimard, «Bibliothèque de la Pléiade », 1991. 
Zola, Émile. Correspondance I. Barend Hendrik Bakker (ed.). Paris et Montréal : CNRS et PU de Montréal, 1978.

\section{NOTES}

1. «Ô fins d'automne, hivers, printemps trempés de boue / Endormeuses saisons! je vous aime et vous loue / D'envelopper ainsi mon cœur et mon cerveau / D'un linceul vaporeux et d'un vague tombeau $(\mathrm{I}, 100)$.

2. William Crookes est un chimiste qui s'intéressa aux phénomènes paranormaux, notamment au cas de Florence Cook, médium qui faisait apparaître l'esprit et se matérialiser le corps de Katie King.

3. À ce sujet, voir Dijkstra 1986 et Dottin-Orsini 1993.

\section{RÉSUMÉS}

Joris-Karl Huysmans définit ainsi le but de sa critique d'art : "surtout décrire le tableau de telle façon que celui qui en lit la traduction écrite, le voie». Autrement dit, les écrits sur l'art huysmansiens visent à rendre visible le tableau décrit, mais ce projet se heurte à un obstacle majeur, celui de la verbalisation d'un phénomène extérieur au langage : la couleur. Aussi, pour évoquer la couleur, Huysmans ne se contente pas d'utiliser ses propres mots: il compare les coloris des artistes au langage d'autres écrivains, s'inscrivant ainsi dans la théorie baudelairienne des correspondances. Le recours à cette analogie signale l'incapacité de la critique d'art en tant que genre à rendre compte de la couleur, et la nécessité qu'elle a de convoquer d'autres textes pour suggérer ce qu'elle échoue à dire.

Defining the aim of his art criticism, Joris-Karl Huysmans wrote : "surtout décrire le tableau de telle façon que celui qui en lit la traduction écrite le voie". In other words, Huysmans, with his writings on art, tends to make visible the painting he describes; thus, he faces a considerable challenge, i.e. verbalising colour, which seems beyond language. To call colour to mind, Huysmans not only uses his own words, but he also compares the colours of artists to the language of other writers, following Baudelaire's "correspondences". The resort to this analogy reveals the inability of art criticism as a genre to depict colour and the necessity to summon other texts to suggest what it fails to tell.

\section{INDEX}

oeuvrecitee À rebours, Croquis parisiens, Écrits sur l'art

Mots-clés : critique d'art, couleur, correspondances

Keywords : art criticism, colour, correspondences 


\section{AUTEURS}

\section{AUDE JEANNEROD}

Aude Jeannerod est docteur en langue et littérature françaises de l'Université Jean Moulin Lyon 3. Elle a soutenu en décembre 2013 sa thèse intitulée « La critique d'art de Joris-Karl Huysmans. Esthétique, poétique, idéologie ». Elle est membre de l'équipe de recherches MARGE (Lyon 3) et de la Société des Études Romantiques et Dix-neuviémistes. Elle contribue à l'édition des CEuvres complètes de Huysmans en préparation aux Classiques Garnier. Site personnel : http:// audejeannerod.wordpress.com 